\title{
Evaluation of trained volunteer doula services for disadvantaged women in five areas in England: women's experiences
}

\begin{tabular}{|r|l|}
\hline Journal: & Health \& Social Care in the Community \\
\hline Manuscript ID & HSCC-OA-15-0197.R1 \\
\hline Manuscript Type: & Original Article \\
\hline Keywords: & $\begin{array}{l}\text { doula, maternity, peer support, social disadvantage, trained volunteer, } \\
\text { non-professional support }\end{array}$ \\
\hline \multicolumn{2}{|l}{} \\
\hline
\end{tabular}

SCHOLARONE $^{\text {m }}$

Manuscripts 
1 Evaluation of trained volunteer doula services for disadvantaged women in five areas in

2 England: women's experiences

3

4

\section{Abstract}

6

7 Disadvantaged childbearing women experience barriers to accessing health and social care

8 services and face greater risk of adverse medical, social and emotional outcomes. Support

9 from doulas (trained lay women) has been identified as a way to improve outcomes; however

10 in the UK doula support is usually paid-for privately by the individual, limiting access among

11 disadvantaged groups. As part of an independent multi-site evaluation of a volunteer doula

12 service this study examined women's experiences of one-to-one support from a trained

13 volunteer doula during pregnancy, labour and the postnatal period among women living in

14 five low-income communities in England.

A mixed methods multi-site evaluation was conducted with women (total $n=137$ ) who received the service before December 2012, using a combination of questionnaires $(n=136)$, and individual or group interviews $(\mathrm{n}=12)$.

Topics explored with women included the timing and nature of support, its impact, the relationship with the doula, and negative experiences. Most women valued volunteer support, describing positive impacts for emotional health and well-being, and their relationships with their partners. Such impacts did not depend upon the volunteer's presence during labour and birth. Indeed, only half $(75 / 137 ; 54.7 \%)$ had a doula attend their birth. Many experienced volunteer support as a friendship, distinct from the relationships offered by healthcare 
26 professionals and family. This led to potential feelings of loss in these often isolated women

27 when the relationship ended.

29 Volunteer doula support that supplements routine maternity services is potentially beneficial

30 for disadvantaged women in the UK even when it does not involve birth support. However,

31 the distress experienced by some women at the conclusion of their relationship with their

32 volunteer doula may compromise the service's impact. Greater consideration is needed for

33 managing the ending of a one-to-one relationship with a volunteer, particularly given the

34 likelihood of it coinciding with a period of heightened emotional vulnerability.

\section{Bullet points}

\section{What is known about this topic}

- Disadvantaged childbearing women are at greater risk of adverse outcomes, partly reflecting barriers to accessing services

- Support from doulas (trained lay women) has been associated with improved outcomes; however doula support is usually paid-for in the UK, limiting access among disadvantaged groups

- Few studies have explored doula support in settings where midwives are the lead health professionals.

\section{What this paper adds}

- Women from low-income communities using a volunteer doula service alongside routine maternity services reported predominantly positive impacts that did not depend upon volunteers attending labour

- Women described feelings of loss when the relationship ended 
51 - Managing the ending of a one-to-one relationship with a volunteer requires greater consideration given its potential to compromise impact.

\section{Introduction}

56 In the UK, most women access maternity care through the National Health Service; this is

57 free at the point of access. Midwives work across hospital and community settings, coordinate the care provided during pregnancy, birth and the early postnatal period and are the lead healthcare professionals for women whose pregnancies are considered low risk.

60 Women may also receive other statutory services e.g. from General Practitioners, health

61 visitors, and social services. Disadvantaged women (including those with complex social

62 needs such as social deprivation, lone parenting, substance misuse, mental illness, domestic

63 abuse, asylum seekers and refugees) are less likely to access routine services and face

64 increased risk of poorer maternal and child health outcomes (Downe et al., 2009, Hodnett et

65 al., 2010, O'hara and Mccabe, 2013, Confidential Enquiry into Maternal and Child Health,

66 2009, Centre for Maternal and Child Enquiries, 2011).

67

68 The National Institute for Health and Care Excellence (NICE) in England and Wales

69 recommended that service provision for pregnant women with complex social needs be better

70 integrated both within the NHS and between the NHS and those services provided in the

71 community by not-for-profit organisations (described in the UK as the voluntary or third

72 sector) (National Institute for Health and Care Excellence, 2010). This fits with a move in

73 high-income countries towards using lay health workers (i.e. those with some training, but no

74 formal professional training or qualification) to engage minority communities and support

75 those with complex needs (Glenton et al., 2013). Recognising the limited evidence base, 
76 NICE identified two research questions that related to this: What effect does involving third

77 [voluntary] sector agencies in providing support and coordination of care for vulnerable

78 women have on outcomes? Is intervention and/or family support provided by statutory and

79 third [voluntary] sector agencies effective in improving outcomes for women and their

80 babies? (National Institute for Health and Care Excellence, 2010).

82 The research reported here examined a voluntary sector service where disadvantaged childbearing women are allocated a volunteer 'doula' (the term adopted by the service) with the aim of enhancing support and wellbeing, and improving the uptake of health and social services. The doulas are volunteers from the local community who receive accredited training, funded by the service; as such they are considered lay rather than professional.

87 Training covers preparation for and support during labour and birth, breastfeeding, child

88 protection, domestic abuse awareness, cultural diversity and communication skills. Salaried

89 service staff match a volunteer (and, sometimes, a back-up volunteer) to each woman

90 according to needs and practicalities; facilitate an initial meeting between the woman and

91 volunteer and mentor the volunteer throughout the support period, typically from the sixth

92 month of pregnancy until six weeks postpartum. Service policy stipulates that doulas and

93 women do not have continued contact beyond the ending of the support period.

95 In common with models of doula support in previously published research (Hodnett et al., 96 2007, Sosa et al., 1980, Steel et al., 2014) the volunteers offer emotional support, 97 information and physical support, but do not provide clinical care. The volunteer doulas 98 differ from traditional schemes in two main ways. Firstly, support extends over a long period 99 rather than being focused on birth and the immediate postpartum; the birth may or may not be 100 attended by the doula. Secondly, the support offered is more diverse and seeks to optimise 
101 women's use of both health and social care services; thus the role includes working closely

102 with existing services, facilitating communications between the woman, her partner and health and social care providers, and signposting to other services, including voluntary and community organisations. In these respects, the closest similar model is the community-based

doulas, an extended doula model which has largely focused on supporting young mothers or those from ethnic minorities (e.g. (Akhavan and Edge, 2012, Breedlove, 2005, Gentry et al., 2010, Wen et al., 2010)). Support in the scheme evaluated here can include: home visits; telephone contact; giving information about services and accompanying to appointments; going for walks and trips to cafes (to reduce social isolation); giving information about pregnancy, labour, birth and looking after the baby; providing physical and emotional support during labour and birth; giving practical help with baby equipment; breastfeeding support.

Previous research has shown doula support to be associated with more positive feelings about labour, increased feelings of control and confidence as a mother and less postnatal depression and anxiety (Gordon et al., 1999, Hofmeyr et al., 1991, Langer et al., 1998, Wolman et al., 1993, Scott et al., 1999). However, research gaps remain. Several studies focused on intrapartum in-hospital support. A recent critical review (Steel et al., 2014) identified the relative absence of research examining the outcomes for women receiving doula support in home or community settings. The review, which focused on 'fee-for-service' doulas, also noted that, despite the focus of doula care being on social and emotional support, research has focused on medical outcomes (i.e. pregnancy and birth outcomes). Alongside the relative dearth of qualitative evidence around recipients' experiences of support is a lack of research into how change is achieved; a notable exception being a grounded theory study identifying the use of several problem-solving strategies used by community-based doulas working with 
125 adolescent mothers (Gentry et al., 2010). In addition, there is a paucity of UK evidence,

126 where doula support is offered alongside midwifery care.

127

128 We conducted an independent multi-site evaluation, informed by Realistic Evaluation

129 (Pawson and Tilley, 1997), which was funded by the National Institute of Health Research.

130 The full report is available (Xxxxxxxx, 2015) [blinded for purpose of peer review]. One of

131 the aims of the evaluation was to examine the health and psychosocial impacts for women

132 who used the volunteer doula service. Analysis of the service databases suggested some

133 clinical outcomes of doula supported women were improved relative to the local population;

134 the caveats around those findings are discussed elsewhere $(\operatorname{Xxxxxxx}, 2015)$ [blinded for

135 purpose of peer review].This paper focuses on the experiences of the women who used the

136 service; specifically, the areas of impact and the nature of the relationship that may offer

137 insights into how such outcomes occur.

Methods

141

142 Settings

143 The doula service was originally set up in site A in 2006 and subsequently in 2011 rolled-out

144 to four other sites (W, X, Y and Z); all of which are low-income communities. The services

145 are predominately run by voluntary sector organisations. Volunteer doula support is provided

146 free of charge to women and is additional to routine statutory and voluntary services. Women

147 may self-refer but are typically referred by another statutory or voluntary agency, usually due

148 to: being unsupported and potentially birthing alone; experiencing health or social problems;

149 or having particular concerns about labour and birth. At two sites services are restricted to 
150 women from ethnic minority groups and a third serves an area with a very large ethnic

151 minority population.

152

153 Ethics and governance

154 Approval for the study was obtained from the West Midlands Research Ethics Committee

155 (reference 12/WM/0342) and governance permissions were obtained at each research site.

157 Eligibility

158 All women who had used the service and whose support had ceased prior to the period of 159 data collection (December 2012-April 2013) were potentially eligible. Exceptions were those

160 whose personal circumstances (for example, stillbirth or certain welfare concerns) meant that 161 contact might increase stress or vulnerability.

163 Procedure

164 Women were invited to complete a questionnaire and/or be interviewed. Questionnaires were 165 completed with the assistance of a researcher or interpreter (by telephone) or self-completed 166 (by post). Interpreter services were favoured over written translation due to the large number 167 of languages used and because service staff indicated that literacy barriers were not limited to 168 English language.

170 Service staff approached women using the recruitment procedure shown in Figure 1 and 171 completed anonymised monitoring logs detailing the dates of contact, reasons for non172 approach and reasons for not sending out research packs. Reminder postcards were sent out 173 three weeks after the initial packs. Women were able to ask questions about the research 174 before deciding whether to participate. All women indicating interest in being interviewed 
175 were provided with further information and written informed consent was secured prior to

176 interview. Interviews were audio-recorded and transcribed.

177

178

179

180

181

182

183

184

185

186

187

188

189

190

191

192

193

194

195

196

197

198

199
[Figure 1 around here]

\section{Development of data collection materials}

Following a Realistic Evaluation perspective, literature review and early discussions with key informants were used to develop topics of interest and a priori hypotheses concerning 'what works for whom, in what circumstances' (Pawson and Tilley, 1997); key informants included service staff and reference panels comprised of volunteer doulas and women who had used the service. The topics and hypotheses were subsequently explored by questionnaire and interviews with participants. No validated questionnaires exist that would enable evaluation of all aspects identified for investigation. A questionnaire was developed and piloted with the women's reference panel. The questionnaire included both open and closed question formats.

Due to length, women using assisted telephone completion were asked a reduced set of questions. A semi-structured interview topic guide was developed, to explore in greater detail women's experiences of some of the issues raised by key informants, including how the volunteer role was similar to and contrasted with support from family, partner and professionals.

Analysis

A mixed methods evaluation was used whereby the method was considered secondary to the research question, reflecting a pragmatic perspective (Johnson et al., 2007, Morgan, 2007). Quantitative questionnaire data were analysed using descriptive statistics and chi-squared 
200 with Yates' continuity correction using SPSS version 20 (Spss Ibm Corp, 2011). Qualitative

201 data (including open-ended questionnaire comments and transcription data) were analysed

202 using content analysis (Grbich, 1999). Anonymous participant identifiers were assigned in

203 the format: data source (Q for questionnaire and I for interview), identification number, study

204 site. The open text questionnaire responses were tabulated to show horizontally all of an

205 individual's responses to the questions and vertically all of the responses received to any

206 question. This facilitated coding of themes on a question-by-question basis, identification of

207 disconfirming responses and the exploration of linked patterns between questions. The

208 transcripts from the interviews were read and reread to gain a detailed familiarity with the

209 overall accounts, and then systematically coded manually both deductively to identify themes

210 related to survey questions and a priori hypotheses and inductively to identify emerging

211 themes (Elo and Kyngäs, 2008). These themes were grouped and collapsed into higher-order

212 conceptual themes with subthemes. The findings of the qualitative and quantitative analyses

213 were integrated to provide a comprehensive narrative of women's experiences.

Impacts presented here include: emotional health and well-being; supporting partners and

women's relationships with their partners; endings and loss. Insights into the nature of the

217 relationship that may inform how these impacts occur are also presented.

\section{Findings}

Questionnaire response rate

223 In total, 627 women had used the service. Of these, $578(92.2 \%)$ were sent a postal

224 questionnaire for self-completion or were contacted by an interpreter or researcher for 
225 assisted telephone completion (see Table 1). Reasons for not making contact or sending the

226 questionnaire were women's circumstances (e.g. stillbirth) and failure to make telephone

227 contact with women who required an interpreter or did not have address details held by the

228 services.

229

230 Questionnaires were completed by 136 women; this represented $21.7 \%$ of women who had

231 used the service. One in eight questionnaires were completed by telephone; the majority

232 using an interpreter (see Table 1). Most women who were interviewed (11/12) also completed

233 a questionnaire.

234

235

[Table 1 around here]

236

237

238 Sample characteristics

239 Sample characteristics were gathered by questionnaire and are reported in Table 2. This was

240 an ethnically diverse sample with 33 countries of birth and 29 main languages listed; 41.0\%

241 did not have English as a main language. Reflecting the service's emphasis on women in

242 situations of disadvantage, including a lack of support, 52.9\% reported not having a

243 supportive partner at the time of the pregnancy and $16.8 \%$ reported having no supportive

244 friends or family at all. Less than half of the women $(40.7 \%)$ were primiparous. Site A's

245 service database indicated that multiparous women and older women were overrepresented

246 amongst questionnaire respondents. The majority of women had been introduced to the

247 service between 2010 and 2012; earlier introductions $(\mathrm{n}=23)$ were limited to the original site,

248 reflecting the service's histories.

249 
[Table 2 around here]

252

253

254

Description of the volunteer support intervention

255 The stages, intensity and nature of volunteer doula support are shown in Table 3. Support in 256 all three stages of the childbearing episode (i.e. antenatal, intrapartum and postnatal support) 257 was most common (47.8\%), followed by support during pregnancy and the postnatal period, 258 without intrapartum support (26.5\%). Of the 122 women whose support commenced during 259 pregnancy, only $75(61.5 \%)$ had their birth attended by a volunteer. This largely reflected 260 women's preferences with just nine women reporting that they had wanted the doula there but 261 that it had not been possible: because the birth happened sooner than anticipated $(n=5)$;

262 because only one birth partner was allowed $(n=3)$; or because the doula was unavailable $263 \quad(n=1)$.

[Table 3 around here]

Impacts of volunteer support

270

271

Impact: Emotional health and well-being

272 The qualitative data illustrated the significance of volunteer support for emotional health and

273 well-being and this was not dependent on the doula being present for labour and birth.

274 Benefits were particularly evident for women with little other support, but were also found 
275 for women who had involved partners or mothers, particularly those women with previous

276 negative experiences of childbearing. Many described the ways in which change occurred,

277 offering insights into mechanisms. The volunteer was someone to talk to and to listen to their

278 concerns in a non-judgemental way, which was important for building confidence and

279 overcoming feelings of isolation, depression, pregnancy worries and birth fears:

280 ... the service should be there for all mothers so won't feel scared or lonely, or ... that's the end of life...I really needed them and they came straight to see me. That's when I saw hope. (Q369Z)

Many women commented on how volunteer support helped them to feel more in control of their maternity care through becoming more aware of their choices; influenced their beliefs in their own physical abilities around birth and confidence for parenting by supporting their choices; and facilitated communication with health professionals, helping to navigate services. Such mechanisms were found both for first-time mothers and mothers who had previously experienced a difficult birth:

291

She was my second voice ... she would say, well we could do this, well we could do do this, or, this is how I'm feeling right now. (I337A) born to get out the house with two babies. (Q334A) 
300 Women's comments illustrated several ways in which doulas had a positive influence on the 301 partner or on the woman-partner relationship through the sharing of roles, alleviating worries 302 and promoting communication. During pregnancy, confiding in a volunteer could mean the 303 woman felt she did not burden her partner with her concerns. Attending the birth could free 304 the woman's partner to care for older children enabling the woman to focus on the birth or 305 the doula could support a partner who also attended (which happened in 36 cases) by 306 explaining things, motivating or reassuring him. Postnatally, the volunteer could help the 307 couple's communication and emotional processing of the birth:

You don't have to worry about looking after him, because you're both just sort of looked after. (I315A)

Impact: Endings and loss

315 The ending of doula support was perceived as a loss for some women. One-third of women $316(n=42 ; 33.1 \%)$ felt that support had ended too soon and often at a difficult time where there were continuing practical or emotional needs:

I had a caesarean section, so somewhat depressed at times. Wish the official time ... should be longer than a mother who had a natural birth. (Q409Y) them and receiving support and then it all stops. (Q332A) 
325 Many spoke of their sadness about the ending of a close relationship. Some felt 'a little

326 discarded' (Q380Y) by this 'temporary friendship' (I337A):

327

328

329

I found it really hard actually, I kept asking if I could keep in touch with her ... but we couldn't... once a friend they become a friend don't they and that's it. (I319A)

330

There was a day she told me that I'm not allowed to get in contact with her, that is not how they do their services, I cried ... oh, I really miss her. (I366Y)

333

334

335

And is not fair according to [service] policy, that when you finish the last day that's it ... She was more than a doula - like family. (Q336X)

336

By contrast, other women found that the support had ended at the right time:

The ending was in the right time, after I felt confident with my baby. (Q408W)

This was particularly likely for women who primarily wanted information from their doula,

343 rather than emotional support, and women at the one site with an extended postnatal support

344 period of three months.

345

346 We hypothesised that endings would be facilitated by having greater preparation. Key

347 informants identified various ways in which doulas prepared women for the ending of the

348 service such as providing an account of their time together or photos. Women for whom a

349 memento had been provided were not less likely to feel that support had ended too soon 
$350\left(31.0 \%\right.$ vs. $\left.37.5 \% ; \chi^{2}=0.24, \mathrm{df}=110, \mathrm{p}=0.63\right)$. The relationship between having something

351 provided and wanting to stay in touch with their volunteer reached borderline significance

$352\left(72.5 \%\right.$ vs. $\left.52.4 \% ; \chi^{2}=3.78, \mathrm{df}=110, \mathrm{p}=0.05\right)$. The finding that mementos did not appear to

353 facilitate endings or reduce feelings of loss may suggest that these acts reflected the quality of

354 the relationship rather than preparation per se.

355

356 Women proposed two ways to improve endings: timing the ending to woman's needs (for

357 example following operative birth), or permitting some contact beyond the ending of support;

358 for example, a one-to-one informal meeting, or a reunion attended by several women and

359 their volunteers. Some women framed this in terms of wanting to be able to thank the

360 volunteer by showing her the long-term impact of her support:

361

362

363

364

365

Just to let her know how I was coping with baby through all her advice. (Q367A)

366

367

368

So I could show her my perfect family because of her and her help. (Q427A)

\section{Understanding the relationship}

Understanding the relationship: How the volunteer is viewed

371 Women were asked to choose all that applied from a list describing how they viewed their

372 volunteer. Most saw her 'as a friend' (88/118; 74.6\%); other answers were 'like a professional' 373 (32.2\%), 'like a family member' (31.4\%; 'like a sister' $21.2 \%$; 'like a mother' $17.8 \%$ ), 'like an 374 advocate' (17.8\%), 'someone like me' (16.9\%), 'like a role model' (14.4\%). 
376 Most of those viewing the volunteer as 'like a family member' had wanted to stay in touch

377 (mother: 90.5\%; sister: 91.7\%; friend 69.0\%; professional 59.5\%). Viewing the doula as like

378 a mother appeared strongly linked to whether that role was missing in the woman's own

379 network. None of the 21 women likening the volunteer to a mother had a supportive mother

380 available during their pregnancy and no-one with a supportive mother described the role in

381 this way. Women with supportive family or friends nonetheless valued their volunteer's

382 support; volunteers were better informed about pregnancy and birth, talked through options

383 and supported the woman's choice in a non-directive way, whereas family and friends may

384 have their own needs and agenda.

385

386 During interview discussions women contrasted volunteer support with health professionals'.

387 They valued the greater accessibility and continuity offered by volunteers, considered 'the

388 one constant person' (I315A). Volunteers were largely viewed as focused completely on the

389 woman ('just there for you', I341Y) with no competing agenda, promoting trust. Many

390 women felt that they could ask their volunteer about anything, including beyond the "medical

391 things' (I486W), whereas they sometimes felt embarrassed or lacked confidence to ask health

392 professionals who were perceived to be busy or dismissive.

393

394 Understanding the relationship: Timing of support

395 We hypothesised that the volunteer support may not 'work' where a match happened late in

396 the antenatal period and there was not time to establish a relationship. One-third (38/115;

$39733.0 \%$ ) felt the relationship would have been different if they had met sooner and $22.6 \%$

$398(26 / 115)$ felt that the relationship would have been different if they had met later. Some

399 women felt that meeting later would not have influenced the relationship because they met 
400 relatively late anyway, just shortly before the birth. Overwhelmingly, women felt that the

401 relationship would have been better for meeting sooner; either to gain the benefits of support

402 earlier in pregnancy or establish the relationship sooner, ensuring the opportunity to develop

403 'trust' (Q332A), get to know each other (Q448A) and 'bond' (Q423A). Consistent with this,

404 some women reported feeling less comfortable with the back-up doula because of lacking the

405 opportunity to develop a relationship.

406

407 Negative experiences

408 A small proportion of women reported negative experiences. Fifteen out of 129 women

$409(11.6 \%)$ reported that the service had not helped them in the way they had hoped. Rating their

410 experience of support from zero (very poor) to five (very good), $11.4 \%(15 / 132)$ rated at

411 three and $2.3 \%(3 / 132)$ rated less than three. Most commonly it was the volunteer's

412 unreliability or inability to provide continuity that was criticised. Some women had been

413 disappointed at the limitations of the service (for example, not assisting with household

414 chores or providing care for older children) and some felt inhibited about asking for more

415 support, knowing that volunteers were unpaid. Indeed, several women, including those

416 reporting positive experiences overall, expressed feelings of guilt about accepting support

417 from a volunteer without the ability to reciprocate.

419 Discussion

420 Most women reported positive impacts on their emotional well-being; including combating

421 feelings of depression, having fears allayed, and building confidence and self-esteem. Whilst

422 similar benefits have been reported elsewhere (Gordon et al., 1999, Hofmeyr et al., 1991,

423 Langer et al., 1998, Scott et al., 1999, Wolman et al., 1993), a key finding of this study is that

424 such benefits did not depend upon doulas being involved in the labour and birth. Benefits 
425 appeared to be achieved through listening by someone who was non-judgemental and non-

426 directive, relief of isolation, information provision, supporting women's choices and help

427 navigating statutory and other services. These findings resonate with Gentry and colleagues

428 (2010) who through interviewing adolescent mothers supported by community-based doulas

429 identified the use of problem-solving strategies including active listening, assuring, affirming,

430 advising and advocating,

431

432 Women also described the mechanisms by which woman-partner relationships were

433 strengthened; including through the sharing of roles, alleviating concerns and promoting

434 communication. The need to involve fathers in pregnancy, childbirth and the transition to

435 parenthood is increasingly recognised by national UK and international policy (Steen et al.,

436 2012). The current research suggests that volunteer doula services may offer a route to

437 supporting involvement, consistent with reports of the Ounce Home Visiting and Doula

438 Program in the US (The Ounce, 2014). Research is needed on perceptions of doula support

439 from the perspectives of partners and other family members (Steel et al., 2014) and how these

440 family relationships may interact with the impacts of the support (Wen et al., 2010)

441

442 Few women reported negative experiences or dissatisfaction although we recognise that this

443 may partly reflect self-selection sampling bias and that women are often reluctant to be

444 critical of their care (Green, 2012). Whilst there were instances of disappointment with the

445 lack of assistance with household chores, as has been reported with lay workers in the context

446 of health visiting (Mackenzie, 2006), dissatisfaction was mainly related to perceiving the

447 volunteer as unreliable or not having as much contact with the volunteers as they wished;

448 something that women felt was harder to negotiate when support was delivered by a

449 volunteer. 
451 Understanding how women viewed their volunteers offered insights into how support 452 'worked', from a theoretical perspective (Pawson and Tilley, 1997). Women frequently

453 likened the volunteer to a family member or friend, consistent with the literature on

454 volunteers and lay workers in the context of childbearing (Hazard et al., 2009, Meier et al., 455 2007, Perkins and Macfarlane, 2001, Taggart et al., 2000, Gentry et al., 2010). Friendship

456 was a central theme here and we note the overlaps between the current volunteer role and 457 other community-based support programmes, such as those that use volunteer befrienders for 458 women who may find it difficult to access or engage with services (Coe and Barlow, 2013).

459 For some women however the concept of friendship was challenged by the unidirectional and 460 unbalanced nature of this relationship; an observation lacking in the doula literature.

462 Few studies have explored doula support in settings where the midwife is the lead health 463 professional. Here, support from volunteers was contrasted with health professionals' with 464 distinctive features of doula support being continuity, not feeling time pressured, feeling able 465 to 'ask anything', feeling their choices were supported and seeing the doulas as more 466 reliable and trustworthy. These findings resonate with studies of lay support for 467 disadvantaged childbearing women in high-income countries; including, community-based 468 doulas in the USA (Gentry et al., 2010), home visits in Australia (Taggart et al., 2000) and 469 the USA (Sheppard et al., 2004) and infant feeding support in the UK (Beake et al., 2005).

470 The greater continuity afforded by doulas compared with midwives has been reported 471 elsewhere in a Swedish study (Lundgren, 2010).

472

473 While participants were largely favourable towards the volunteer doula support and valued 474 the continuity provided, it was striking that women commonly reported feelings of loss 
475 around the ending of support, which could constitute a negative impact. Volunteer support

476 was valued regardless of whether women had support from their friends or family. The aspect

477 of support often valued most highly was the one-to-one relationship. Its ending could be

478 particularly difficult for some women, particularly those who viewed the volunteer as like a

479 mother or where there were continuing practical needs, for instance, following an operative

480 birth. Even women who felt well-prepared to move on independently and did not have

481 continuing support needs could still feel saddened by the absence of opportunity for any

482 contact with the volunteer in the future.

483

484 These findings highlight the challenges noted elsewhere in the volunteer and lay worker

485 literature around ways of working that hinge on a close relationship between worker and

486 recipient and the need to consider further the management of emotional relationships and

487 boundaries (Glenton et al., 2013, Heslop, 2006, Mitchell and Pistrang, 2011, Gillard et al.,

488 2014, Perkins and Macfarlane, 2001, Simpson et al., 2014). These challenges are not limited

489 to relationships with volunteer and lay workers. Similar experiences have been reported with

490 caseload midwifery with women reporting 'midwife grief' and feeling lost or abandoned at

491 the end of the period of support (Walsh, 1999).

492

493 It is feasible that such endings may compromise the impact of the period of support. In social

494 work, concerns have been expressed that endings may reinforce previous negative separation

495 experiences (Huntley, 2002). In psychotherapy it is recognised that abrupt endings and forced

496 endings have the potential to be harmful (Gelso and Woodhouse, 2002). A recent systematic

497 review of befriending in mental health (Thompson et al., 2015) argued that experiencing

498 some of the qualities of friendship accompanied by an enforced ending could lead to the 
intervention failing, calling for clearer expectations for support recipients about the nature of

500 what is being offered.

501

502 Continuing doula support beyond six weeks postpartum should be considered, especially

503 since this coincides with a time of peak incidence of postnatal depression (Cox et al., 1993).

504 There was some indication that endings may have been easier at the one site where postnatal

505 contact extended until 12 weeks after birth although sample sizes precluded definitive

506 comparisons. Regardless of the length of postnatal support, the ending itself still requires

507 planning and appropriate management, with support from service staff, as required. Several

508 women suggested changing the service to offer an informal meeting to provide an update,

509 group-based, if necessary. Other evaluations of peer support have recommended using more

510 teamwork, using goals and being problem-focused to minimise dependency in a one-to-one

511 relationship (Perkins and Macfarlane, 2001, Repper and Watson, 2012); such ways of

512 working may help to enable a transition from the one-to-one relationship but it is unknown

513 how this would influence the impact of support.

515 Strengths and Limitations

516 This is the largest independent evaluation of trained volunteer doula support in the UK and

517 our findings reflect those of another independent evaluation of one doula service (Granville

518 and Sugarman, 2012). Questionnaire data were complemented by interviews, which offered

519 opportunities for more detailed exploration, including the ways in which the volunteer role

520 was similar to and contrasted with support from family, partner and professionals. A strength

521 of our evaluation was the representation of women of non-English speaking background;

522 however the questionnaire was only completed by $21.7 \%$ of women who had used the

523 service, posing some concerns around sampling bias and transferability of findings. A low 
524 response was anticipated because support recipients were in situations of disadvantage with

525 high mobility and in groups traditionally hard to engage in research. In addition, some

526 recipients had accessed the service several years previously and could no longer be contacted.

527 It was not possible to determine from the information provided by the services the extent to

528 which participants were representative in terms of time since using the service and we

529 acknowledge that there is potential impact for memory bias that was not explored here. A

530 higher response rate would be necessary to explore fully the influence of the ending of the

531 relationship on the overall impact of a volunteer doula service.

532

533 Efforts to maximise responding included approach via a known service (also essential due to

534 confidentiality) and assisted questionnaire completion. However any positive impacts from

535 these efforts was possibly limited by language needs being under-recognised by the services,

536 who documented the need for an interpreter, rather than the main language(s) spoken and it

537 appeared that some women may have been sent written information that did not meet their

538 language needs. Unfortunately, fewer data were available for those women using assisted

539 completion because of the need to ensure that the questionnaire length remained acceptable.

540

541 Conclusion

542 The UK NICE guidance for the care of Pregnant women with Complex Social Factors

543 (National Institute for Health and Care Excellence, 2010) calls for models that overcome

544 barriers and facilitate access to improve women's outcomes. It would appear that volunteer

545 doula services have the potential to make a contribution to this. Of note, the benefits reported

546 by women did not always involve direct support during the labour and birth. An approach

547 akin to friendship and based on building trust, listening and enabling appears to be

548 fundamental; in some circumstances this can be strengthened by actively supporting 
549 involvement of family, including partners. Critically, the ending of the close one-to-one

550 relationship carries the potential for feelings of loss and distress which could undermine the

551 benefits experienced. The timing and management of endings warrant further exploration,

552 particularly given the potential for coinciding with a period of heightened vulnerability for

553 mental health problems, Further longitudinal research is needed to gather women's views and

554 experiences through the period of support, and the ending, to further elucidate the

555 mechanisms by which positive impacts of doula support are achieved and may be threatened.

556

557 References

558 Akhavan, S. \& Edge, D. 2012. Foreign-Born Women's Experiences of Community-Based

559 Doulas in Sweden - A Qualitative Study. Health Care for Women International, 33,

$560 \quad 833-848$.

561 Beake, S., Mccourt, C., Rowan, C. \& Taylor, J. 2005. Evaluation of the use of health care

562 assistants to support disadvantaged women breastfeeding in the community. Matern

$563 \quad$ Child Nutr, 1, 32-43.

564 Breedlove, G. 2005. Perceptions of social support from pregnant and parenting adolescents

565 using community-based doulas. The Journal of Perinatal Education, 14, 15-22.

566 Centre for Maternal and Child Enquiries 2011. Saving Mothers' Lives: Reviewing maternal

567 deaths to make motherhood safer: 2006-2008. The Eighth Report of the Confidential

568 Enquiries into Maternal Deaths in the United Kingdom. BJOG, 118, 1-203.

569 Coe, C. \& Barlow, J. 2013. Supporting women with perinatal mental health problems: the

570 role of the voluntary sector. Community Pract, 86, 23-27.

571 Confidential Enquiry into Maternal and Child Health 2009. Perinatal Mortality 2009.

572 London: CEMACH. 
573 Cox, J. L., Murray, D. \& Chapman, G. 1993. A controlled study of the onset, duration and 574 prevalence of postnatal depression. Br J Psychiatry, 163, 27-31.

575 Downe, S., Finlayson, K., Walsh, D. \& Lavender, T. 2009. 'Weighing up and balancing out':

576 a metasynthesis of barriers to antenatal care for marginalised women in high-income

$577 \quad$ countries. BJOG, 116, 518-529.

578 Elo, S. \& Kyngäs, H. 2008. The qualitative content analysis process. Journal of Advanced $579 \quad$ Nursing, 62, 107-115.

580 Gelso, C. J. \& Woodhouse, S. S. 2002. The termination of psychotherapy: What research tells 581 us about the process of ending treatment. In: TRYON, G. S. (ed.) Counseling based 582 on process research: Applying what we know. Boston, MA: Allyn \& Bacon. 583 Gentry, Q. M., Nolte, K. M., Gonzalez, A., Pearson, M. \& Ivey, S. 2010. “Going Beyond the 584 Call of Doula": A Grounded Theory Analysis of the Diverse Roles Community585 Based Doulas Play in the Lives of Pregnant and Parenting Adolescent Mothers. The $586 \quad$ Journal of Perinatal Education, 19, 24-40.

587 Gillard, S., Edwards, C., Gibson, S., Holley, J. \& Owen, K. 2014. New ways of working in 588 mental health services: A qualitative, comparative case study assessing and informing 589 the emergence of new peer worker roles in mental health services in England. Health $590 \quad$ Services and Delivery Research, 2, 218.

591 Glenton, C., Colvin, C. J., Carlsen, B., Swartz, A., Lewin, S., Noyes, J. \& Rashidian, A. 592 2013. Barriers and facilitators to the implementation of lay health worker programmes 593 to improve access to maternal and child health: qualitative evidence synthesis. $594 \quad$ Cochrane Database Syst Rev, 10.

595 Gordon, N. P., Walton, D., Mcadam, E., Derman, J., Gallitero, G. \& Garrett, L. 1999. Effects 596 of providing hospital-based doulas in health maintenance organization hospitals. $597 \quad$ Obstet Gynaecol, 93, 422-6. 
598 Granville, G. \& Sugarman, W. 2012. Parents 1st Independent Evaluation. "Someone in my 599 corner": A volunteer peer support programme for parenthood, birth and beyond. Final $600 \quad$ Evaluation Report: November 2012.: Gillian Granville Associates.

601 Grbich, C. 1999. Qualitative Research in Health: An introduction, London, Sage.

602 Green, J. 2012. Integrating women's views into maternity care research and practice. Birth, $603 \quad 39,291-295$.

604 Hazard, C. J., Callister, L. C., Birkhead, A. \& Nichols, L. 2009. Hispanic Labor Friends 605 Initiative: supporting vulnerable women. Am J Matern Child Nurs, 34, 115-121. 606 Heslop, P. 2006. Good practice in befriending services for people with learning difficulties. 607 BrJ Learn Disabil, 33, 27-33.

608 Hodnett, E. D., Fredericks, S. \& Weston, J. 2010. Support during pregnancy for women at 609 increased risk of low birthweight babies. Cochrane Database Syst Rev, CD000198. 610 Hodnett, E. D., Gates, S., Hofmeyr, G. J. \& Sakala, C. 2007. Continuous support for women 611 during childbirth. Cochrane Database Syst Rev, 3, CD003766.

612 Hofmeyr, G. J., Nikodem, V. C., Wolman, W. L., Chalmers, B. E. \& Kramer, T. 1991. 613 Companionship to modify the clinical birth environment: effects on progress and 614 perceptions of labour, and breastfeeding. BJOG, 98, 756-64.

615 Huntley, M. 2002. Relationship based social work - how do endings impact on the client? $616 \quad$ Practice, 14, 59-66.

617 Johnson, R. B., Onwuegbuzie, A. J. \& Turner, L. A. 2007. Toward a definition of mixed 618 methods research. Journal of Mixed Methods Research, 1, 112-133.

619 Langer, A., Campero, L., Garcia, C. \& Reynoso, S. 1998. Effects of psychosocial support 620 during labour and childbirth on breastfeeding, medical interventions, and mothers' 621 wellbeing in a Mexican public hospital: a randomised clinical trial. BJOG, 105, 1056622 63. 
623 Lundgren, I. 2010. Swedish women's experiences of doula support during childbirth. $624 \quad$ Midwifery, 26, 173-180.

625 Mackenzie, M. 2006. Benefit or burden: introducing paraprofessional support staff to health 626 visiting teams: the case of Starting Well. Health Soc Care Community, 14, 523-531.

627 Meier, E. R., Olson, B. H., Benton, P., Eghtedary, K. \& Song, W. O. 2007. A qualitative 628 evaluation of a breastfeeding peer counselor program. J Hum Lact, 23, 262-268.

629 Mitchell, G. \& Pistrang, N. 2011. Befriending for mental health problems: Processes of 630 helping. Psychol Psychother, 84, 151-169.

631 Morgan, D. L. 2007. Paradigms lost and pragmatism regained: Methodological implications 632 of combining qualitative and quantitative research. Journal of Mixed Methods $633 \quad$ Research, 1, 48-76.

634 National Institute for Health and Care Excellence 2010. Pregnancy and complex social 635 factors: A model for service provision for pregnant women with complex social 636 factors. Nice Clinical Guideline 110. London: National Institute for Health and Care 637 Excellence.

638 O'hara, M. W. \& Mccabe, J. E. 2013. Postpartum Depression: Current Status and Future 639 Directions. Annu Rev Clin Psychol, 9, 379-407.

640 Pawson, R. \& Tilley, N. 1997. Realistic Evaluation, London, Sage.

641 Perkins, E. R. \& Macfarlane, J. 2001. Family support by lay workers: a health visiting 642 initiative. Br J Community Nurs, 6, 26-32.

643 Repper, J. \& Watson, E. 2012. A year of peer support in Nottingham: lessons learned. 644 Journal of Mental Health Training, Education and Practice, 7, 70-78.

645 Scott, K. D., Klaus, P. H. \& Klaus, M. H. 1999. The Obstetrical and Postpartum Benefits of 646 Continuous Support during Childbirth. $J$ Womens Health Gend Based Med, 8, 1257647 1264. 
648 Sheppard, V. B., Williams, K. P. \& Richardson, J. T. 2004. Women's priorities for lay health 649 home visitors: implications for eliminating health disparities among underserved 650 women. J Health Soc Policy, 18, 19-35.

651 Simpson, A., Quigley, J., Henry, S. \& Hall, C. 2014. Evaluating the selection, training, and 652 support of peer support workers in the United Kingdom. Journal of Psychosocial Nursing and Mental Health Services, 52, 31-40.

Sosa, R., Kennell, J., Klaus, M., Robertson, S. \& Urrutia, J. 1980. The effect of a supportive companion on perinatal problems, length of labor, and mother-infant interaction. New Engl J Med, 303, 597-600.

Spss Ibm Corp 2011. IBM SPSS Statistics for Windows, Version 20.0., Armonk, NY, IBM Corp.

Steel, A., Frawley, J., Adams, J. \& Diezel, H. 2014. Trained or professional doulas in the support and care of pregnant and birthing women: a critical integrative review. Health Soc Care Community, early view online, 17.

Steen, M., Downe, S., Bamford, N. \& Edozien, L. 2012. Not-patient and not-visitor: A metasynthesis of fathers' encounters with pregnancy, birth and maternity care. Midwifery, 28, 422-431.

Taggart, A. V., Short, S. D. \& Barclay, L. 2000. 'She has made me feel human again': an evaluation of a volunteer home-based visiting project for mothers. Health Soc Care Community, 8, 1-8.

The Ounce 2014. Fiscal Year 2013 Ounce Home Visiting and Doula Programs Annual Report. Chicago, Illinois: Ounce of Prevention Fund.

Thompson, R., Valenti, E., Siette, J. \& S., P. 2015. To befriend or to be a friend: a systematic review of the meaning and practice of "befriending" in mental health care. Journal of Mental Health, Early Online, 1-7. 
673 Walsh, D. 1999. An ethnographic study of women's experience of partnership caseload

674 midwifery practice: The professional as a friend. Midwifery, 15, 165-176.

675 Wen, X., Korfmacher, J., Hans, S. L. \& Henson, L. G. 2010. Young mothers' involvement in

676 a prenatal and postpartum support program. Journal of Community Psychology, 38,

$677 \quad 172-190$.

678 Wolman, W. L., Chalmers, B., Hofmeyr, G. J. \& Nikodem, V. C. 1993. Postpartum

679 depression and companionship in the clinical birth environment: a randomized,

680 controlled study. Am J Obstet Gynecol, 168, 1388-93.

681 Xxxxxxxx 2015. details not provided for purpose of double blind review process.

682

683 


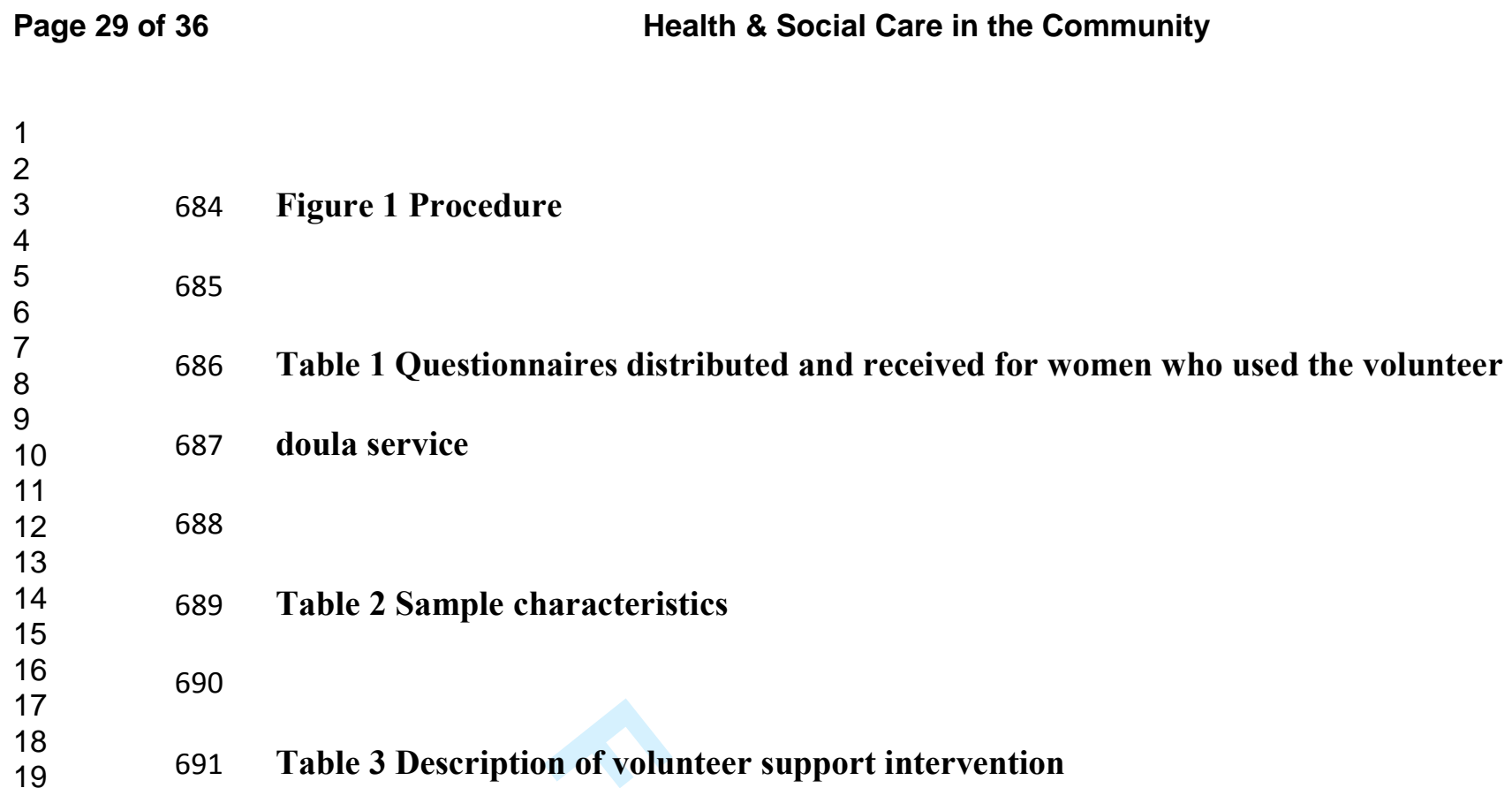


1 Table 1 Questionnaires distributed and received for women who used the volunteer doula service

2

\begin{tabular}{|c|c|c|c|c|c|c|c|c|}
\hline Study & Women & Sent or & Self-completion & Assisted & Assisted & Total & Percentage of & Response rate \\
\hline \multirow[t]{3}{*}{ Site } & supported by & approached by & & completion & completion & completed (any & those supported & of those \\
\hline & the service & interpreter/ & & with interpreter & with researcher & method) & by the service & approached (\%) \\
\hline & & researcher & & & & & $(\%)$ & \\
\hline $\mathrm{A}$ & 446 & 417 & 83 & 7 & 0 & 90 & 20.2 & 21.6 \\
\hline W & 51 & 50 & 13 & 1 & 0 & 14 & 27.5 & 28.0 \\
\hline $\mathrm{X}$ & 29 & 26 & 8 & 1 & 0 & 9 & 31.0 & 34.6 \\
\hline Y & 75 & 68 & 14 & 0 & 0 & 14 & 18.7 & 20.6 \\
\hline $\mathrm{Z}$ & 26 & 17 & 1 & 6 & 2 & 9 & 34.6 & 52.9 \\
\hline Total & 627 & 578 & 119 & 15 & 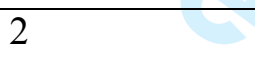 & 136 & 21.7 & 23.5 \\
\hline
\end{tabular}

3 
1 Table 2 Sample characteristics

2

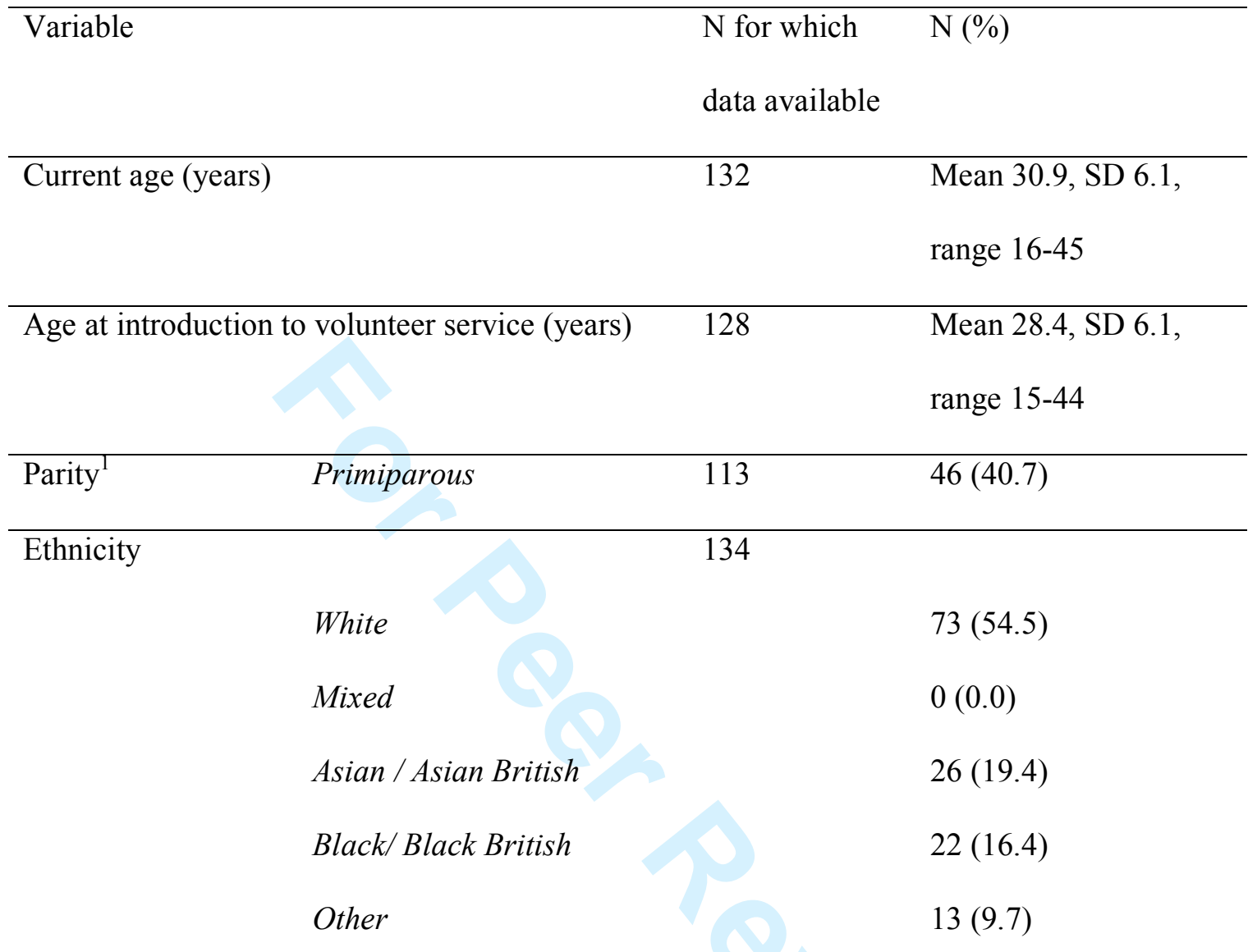

Time in UK at introduction to doula service 130

Since birth

$66(50.8)$

$>5$ years

$20(15.4)$

$1-5$ years

$30(23.1)$

$<1$ year

$14(10.8)$

Main language

134

English

$73(54.5)$

English and another

$6(4.5)$

non-English

$55(41.0)$

Age left school or college (years)

119

59

60 
119

partner/husband

$56(47.1)$

other

none
$20(16.8)$

\begin{tabular}{lll}
\hline Social complexity $^{2}$ & 136 & $46(33.8)$
\end{tabular}

3 Notes: ${ }^{1}$ Variables that were omitted from the assisted completion questionnaires, due to

4 length. ${ }^{2}$ Social complexity was derived from coding services in contact with women at time

5 of introduction to service, based on descriptions given in the guidance on women with

6 complex social factors (National Institute for Health and Care Excellence, 2010). 
1 Table 3 Description of volunteer support intervention

2

\begin{tabular}{ll}
\hline Variable & $\mathrm{N}$ for which \\
& $\mathrm{N}(\%)$ \\
& data available
\end{tabular}

Stages of support 136

Antenatal only

$16(11.8)$

Antenatal and intrapartum

Antenatal and postnatal

$36(26.5)$

Intrapartum only

Intrapartum and postnatal

Postnatal only

$9(6.6)$

All three stages

$65(47.8)$

Intensity of support (hours per week) 98

Median 2.0, IQR 1.5,

range $0-10$

Antenatal support behaviours

121

Home visits

$106(87.6)$

Telephone support

79 (65.3)

Information giving

87 (71.9)

Birth preparation

$85(70.2)$

Practical help with baby equipment

$51(42.1)$

Came to health/other appointments

$51(42.1)$

Help find out about other services

$66(54.5)$

Go for walks, trips to café etc

$41(33.9)$

Postnatal support behaviours

112

Home visits

$104(92.9)$ 
$\begin{array}{ll}\text { Telephone support } & 62(55.4)\end{array}$

$\begin{array}{ll}\text { Information giving } & 45(40.2)\end{array}$

$\begin{array}{lr}\text { Breastfeeding support } & 56(50.0)\end{array}$

$\begin{array}{ll}\text { Practical help with baby equipment } & 31 \text { (27.7) }\end{array}$

Came to health/other appointments 21 (18.8)

Help find out about other services $\quad 39$ (34.8)

Go for walks, trips to café etc $20(17.9)$

Contact with a back-up volunteer

$\begin{array}{lll}\text { Allocated a back-up } & 119 & 52(43.7)\end{array}$

Type of visits with a back-up volunteer 51

Back-up attended one joint visit $\quad 12$ (23.5)

Back-up attended more than one joint visit 20 (39.2)

Back-up made separate visits 2 (3.9)

\begin{tabular}{ll}
\hline Preparation for ending & 115
\end{tabular}

$\begin{array}{ll}\text { Prepared something (any) } & 71(61.7)\end{array}$

$\begin{array}{ll}\text { Prepared account of time together } & 31(27.0)\end{array}$

$\begin{array}{ll}\text { Prepared photographs } & 30(26.1)\end{array}$

Prepared birth story $23(20.0)$

3 Notes: IQR = inter-quartile range

4

5 
Service staff telephone woman using last known contact details (using bilingual staff or interpreter where required, as indicated by service records)
No reply

(following three attempts by call or text message)

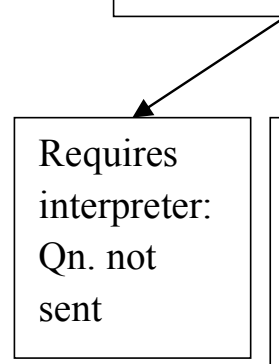

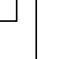

\begin{tabular}{|l|l|}
\hline Interpreter & Woman \\
not & declines: \\
required: & Qn. not \\
Qn. and & SAE sent \\
sent
\end{tabular}

for selfcompletion

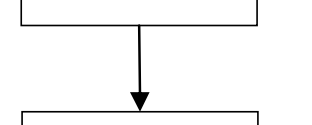

\section{Speaks with woman and introduces the} research; i) asks willingness for research pack to be sent or offers assisted completion; ii) asks willingness for research team to make contact about group interviews

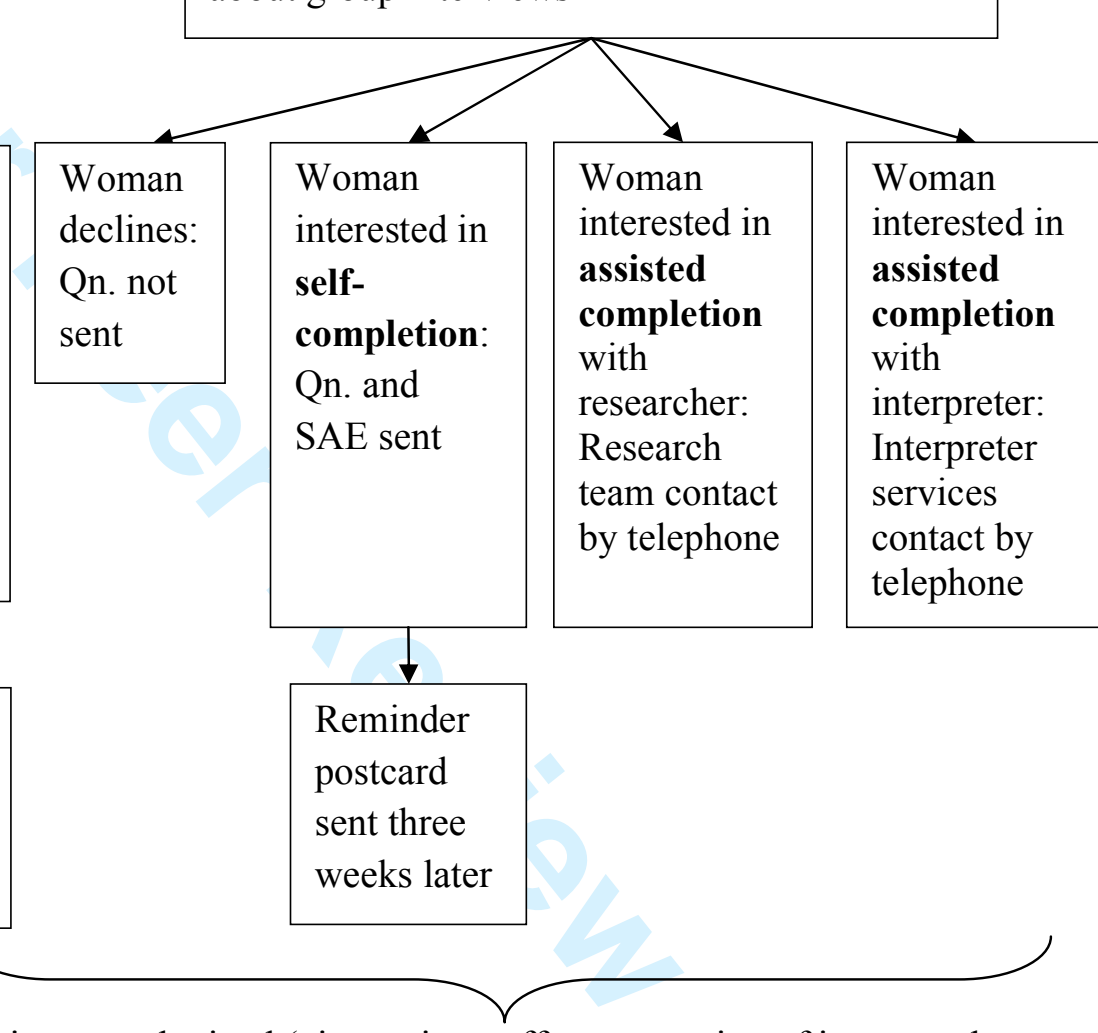

Where permission was obtained (via service staff or expression of interest when completing questionnaire), research team contacted women directly to provide further details about group interviews.

Interviews were held in community venues identified by service staff and were audio-recorded.

Shopping voucher sent to woman on receipt of completed questionnaire and/or provided at attendance of group interview, to thank for participation.

Qn. = questionnaire

SAE = stamped addressed envelope, returned directly to research team 
